\title{
Frontières
}

\section{Prévenir plutôt que partir en peur}

\section{Diane Laflamme}

Volume 21, numéro 1, automne 2008

Prévenir le suicide

URI : https://id.erudit.org/iderudit/037867ar

DOI : https://doi.org/10.7202/037867ar

Aller au sommaire du numéro

Éditeur(s)

Université du Québec à Montréal

ISSN

1180-3479 (imprimé)

1916-0976 (numérique)

Découvrir la revue

Citer ce document

Laflamme, D. (2008). Prévenir plutôt que partir en peur. Frontières, 21(1), 3-7. https://doi.org/10.7202/037867ar d'utilisation que vous pouvez consulter en ligne.

https://apropos.erudit.org/fr/usagers/politique-dutilisation/ 


$\begin{array}{llllllll}D & \text { I } & \mathrm{T} & \mathrm{O} & \mathrm{R} & \mathrm{I} & \mathrm{A} & \mathrm{L}\end{array}$

\section{Prévenir plutôt que partir en peur}

[...]

MAIS LE CEUR DES VIVANTS EST DUR COMME UN BON ARBRE

ET ILS S'EN VONT FORTS DE LEUR VIE

POURTANT LE CEUR DES MORTS EST DÉJÀ TOUT EN SANG

ET OCCUPÉ D'ANGOISSE DEPUIS LONGTEMPS

ET TOUT EN PROIE AUX COUPS, TROP ACCESSIBLE AUX COUPS

À TRAVERS LEUR CARCASSE OUVERTE

MAIS LES VIVANTS PASSANT N'ONT PAS PITIÉ DES MORTS

QUI RESTENT AVEC LEUR CCEUR AU VENT SANS ABRI.

$[\ldots]$

AH! DANS QUEL DÉSERT FAUT-IL QU'ON S'EN AILLE

POUR MOURIR DE SOI-MÊME TRANQUILLEMENT.

SAINT-DENYS-GARNEAU (1949).

POÉSIES COMPLĖTES, «LA MORT GRANDISSANTE », P. 202, 201.

\section{Diane Laflamme, Ph. D.,} rédactrice en chef.

Partir en peur ${ }^{1}$ ? Qui oserait m'en blâmer si ce que je vis et veux fuir à tout prix est une explosion dont l'intensité tient du feu nucléaire ${ }^{2}$, quelque chose qui brûle à la fois autour de moi et en moi, comme une Ignition interne pour reprendre le titre de l'œuvre de l'artiste québécois Pascal Huot, qui figure en page couverture de ce numéro sur la prévention du suicide. Nous savons fort bien chacune et chacun à notre façon, chacune et chacun selon nos blessures que trop souvent la vie est un «Théâtre funeste» - et c'est d'ailleurs sous ce titre que l'artiste a regroupé la plupart des œuvres présentées ici dans nos pages. Nous le savons et si nous sommes là pour en deviser tranquillement, c'est que, tant bien que mal, nous réussissons à nous en accommoder.
Certains d'entre nous - peut-être ceux dont le cœur est «trop accessible aux coups» dont parle Saint-Denys-Garneau dans l'extrait cité en exergue - choisissent plutôt d'avancer l'échéance, de précipiter le dénouement de la tragédie, nous mettant ainsi en présence d'un phénomène qui nous effraie.

Peur: pour moi, c'est le premier vocable qui s'associe d'emblée au mot suicide. Il se peut qu'il en soit autrement pour bien des lecteurs de Frontières. Peut-être, aussi, suis-je la seule à trembler en regardant l'œuvre qui accompagne cet éditorial. Elle s'intitule Silence, et l'original - sur fond rose tendre - me ramène à toute mon impuissance car il me dit, car il me montre la vulnérabilité, la fragilité de celui, de celle parmi tous ceux que j'aime qui pourrait refuser une vie qui lui fait trop mal et choisir l'irréparable, choisir de se donner la mort. 
Prendre l'épouvante, n'est-ce pas là une solution toute trouvée pour les observateurs externes d'une telle tragédie: pour moi et probablement aussi pour les chercheurs et les praticiens qui voudraient développer une réflexion et mettre en place des cadres d'intervention pour prévenir le suicide? Au fil de ses numéros et grâce à la contribution d'auteurs provenant de divers champs disciplinaires, Frontières a proposé à ses lecteurs des résultats de recherche, des points de vue, des témoignages qui ont contribué à dire la complexité de ce phénomène et qui nous donnent des outils pour éviter de partir en peur quand il est question de suicide. Depuis vingt ans, Frontières accompagne la démarche des chercheurs et des intervenants, certes, mais elle ouvre aussi ses pages aux témoignages des écrivains, aux analyses littéraires et au regard du poète sur des thèmes difficiles comme celui du suicide. Déjà lors de sa première année de publication, en 1988, Frontières choisissait le suicide comme thème de son deuxième numéro intitulé : "Le suicide. Vertige aux confins... »³. Une décennie plus tard, le volume 12 a repris ce thème sous le titre: "Suicides, générations et culture ${ }^{4}$. C'est dans cette tradition d'une réflexion multidisciplinaire que s'inscrit le présent numéro intitulé «Prévenir le suicide», qui paraît au moment même où l'Institut national de santé publique du Québec vient de publier des données indiquant enfin un recul de la mortalité par suicide au Québec 5 .

Depuis la parution du numéro thématique de 1999, plusieurs autres articles portant sur le suicide ont paru dans nos pages, ramenant cette problématique à l'occasion d'une réflexion sur un thème complémentaire. En plus de me référer à quelques auteurs du présent numéro, je puiserai surtout dans ces textes publiés dans des numéros antérieurs de la revue afin de montrer comment «partir en peur» quand il est question de suicide, ce serait nier le tragique de l'attestation de soi et aussi oublier la complémentarité des disciplines qui éclairent l'intervention visant à prévenir le suicide.

\section{PARTIR EN PEUR, CE SERAIT NIER \\ LE TRAGIQUE DE L'ATTESTATION DE SOI}

J'emprunte la notion d'attestation de soi au philosophe Paul Ricoeur, qui lui donne une place importante dans son analyse de l'identité personnelle, de l'identité narrative et de la capacité éthique. Pour Ricoeur, l'attestation «peut se définir comme l'assurance d'être soi-même agissant et souffrant » $(1990$, p. 35; 1991, p. 382). Cette certitude de soi ne peut provenir que du soi lui-même: lui seul a la capacité de se la donner et, paradoxalement, il ne peut se la donner qu'en la recevant aussi d'autrui à travers le quotidien d'un vécu, d'une expérience, du récit d'une vie qui deviennent autant de façons de dire à autrui et au monde, «me voici!» :

Ce sera un des leitmotive de notre analyse : l'attestation est fondamentalement attestation de soi. Cette confiance sera tour à tour confiance dans le pouvoir de dire, dans le pouvoir de faire, dans le pouvoir de se reconnaître personnage de récit, dans le pouvoir enfin de répondre à l'accusation par l'accusatif: me voici! (Ricoeur, 1990, p. 34)

L'attestation peut prendre diverses formes selon qu'elle s'actualise dans ce que je dis, dans ce que je fais, dans ce que je raconte à mon sujet ou dans ma façon de rendre compte des actes que je choisis de poser, les deux derniers éléments de cette énumération m'obligeant - il faut le noter - à une observation des deux premiers.

L'attestation de soi réussira-t-elle à garder la mort en échec? Elle est la réponse que je répète, que je confirme au fil des jours, que j'oppose à la possibilité toujours présente, à la possibilité «absolument certaine» de ma mort. Emmanuel Lévinas, un philosophe qui, comme Ricoeur, s'inscrit dans la tradition phénoménologique, écrit:
La mort n'est pas alors la finition d'une durée faite de jours et de nuits, mais une possibilité toujours ouverte. Possibilité toujours ouverte qui est possibilité la plus propre, exclusive d'autrui, isolante, et possibilité extrême ou indépassable.

[...]

La mort est une possibilité absolument certaine; elle est la possibilité qui rend possible toute possibilité. (1991, p. 52, 54)

Le suicide nous met en présence d'une figure tragique de l'attestation de soi. Figure et non pas antithèse car l'attestation de soi ne trouve pas son contraire dans le suicide. Ricoeur précise que le contraire de l'attestation, c'est plutôt le soupçon. Et il ajoute à ce égard qu'«il n'y a pas d'autre recours contre le soupçon qu'une attestation plus fiable» (1990, p. 34). C'est là une remarque d'importance majeure pour notre propos qui s'interroge ici sur la prévention du suicide. L'attestation plus fiable qui me permet de dire «me voici!» avec une assurance renouvelée doit constamment passer l'épreuve du soupçon. Ricoeur présente cette position en faisant ressortir le contraste avec les deux notions opposées de l'être vrai et de l'être faux selon Aristote. À un logique binaire qui distingue deux éléments opposés formant une paire et excluant qu'un troisième élément puisse coexister à cette paire - être vrai ou être faux, rien d'autre -, Ricoeur substitue une logique qui permet d'inclure le tiers précédemment exclu ${ }^{6}$ : le soupçon n'est pas que l'élément opposé de la paire attestation/soupçon, l'un excluant l'autre, il est aussi le chemin vers l'attestation et la traversée dans l'attestation:

Je voudrais marquer par un seul trait différentiel l'écart qui sépare l'être-vrai selon l'attestation de l'être-vrai selon la métaphysique d'Aristote. L'attestation [...] a pour contraire le soupçon. En ce sens, le soupçon occupe la place de l'être-faux dans la paire aristotélicienne. Mais si le soupçon appartient bien au même plan aléthique que l'attestation donc à un plan à la fois épistémique et ontologique -, il se rapporte à l'attestation d'une façon tout à fait originale. Il n'est pas simplement son contraire, en un sens purement disjonctif, comme l'être-faux l'est par rapport à l'être-vrai. Le soupçon est aussi le chemin vers et la traversée dans l'attestation.» (1990, p. 350)

Répétons, en le modulant selon le contexte qui nous intéresse ici, le constat de Ricoeur: le soupçon qui s'apprête à nier le soi qui tente désespérément d'attester de son existence est en même temps le chemin vers l'attestation, la traversée dans l'attestation. Endormir ou engourdir la capacité de se soupçonner incapable ne résoudra rien.

Ce chemin vers l'attestation et cette traversée dans l'attestation, sur fond de soupçon, de remise en question de soi, pourront s'avérer tragiques. Ricoeur le sait d'expérience; il était aussi un père, un père dont le fils s'est suicidé:

Notre second fils Olivier, l'enfant du retour de captivité ${ }^{7}$, l'enfant de la paix, se donnait la mort, le jour même où j'étais à Prague auprès de nos amis [...] Cette catastrophe devait laisser une plaie ouverte que l'interminable travail de deuil n'a pas encore cicatrisée. Encore maintenant, je suis la proie de deux reproches alternés: l'un de n'avoir pas su dire non au moment opportun à certaines dérives, l'autre de n'avoir pas discerné, ni entendu l'appel au secours lancé du fond de la détresse. Je rejoignais ainsi le lot immense de tant de pères et découvrais cette fraternité silencieuse qui naît de l'égalité dans la souffrance. (Ricoeur, 1995, p. 140)

Dans son ouvrage Soi-même comme un autre, après les chapitres sur l'identité personnelle et l'identité narrative, et immédiatement avant de s'engager dans sa neuvième étude qui porte sur le soi et la sagesse pratique, Ricoeur introduit un «Interlude», imprimé en italique et portant cette dédicace poignante: «pour Olivier encore». Ce court texte s'intitule «Le tragique de l'action». 
Il permettra, dit Ricoeur, «de faire entendre une autre voix que celle de la philosophie», celle de la tragédie ${ }^{8}$. Les traits qu'il retient pour caractériser la tragédie ${ }^{9}$ en la distinguant de la philosophie s'énoncent comme suit:

Tels sont les traits qui marquent le caractère non philo-

sophique de la tragédie: puissances mythiques adverses

doublant les conflits identifiables de rôles; mélange inana-

lysable de contraintes destinales et de choix délibérés; effet

purgatif exercé par le spectacle lui-même au cœur des pas-

sions que celui-ci engendre. (1990, p. 283)

La tragédie nous met en présence de drames éminemment personnels, et pourtant les générations qui se succèdent continuent de se ressourcer inlassablement à même les productions qu'elle inspire et de se reconnaître malgré les masques qui servent à sa mise en scène. La «reconnaissance de soi » est à maints égards un préalable à l'attestation de soi qui, en retour, en nourrit la clarté. Ricoeur précise que la reconnaissance de soi s'apprend et que, parfois, c'est la tragédie qui l'enseigne: «Et pourtant la tragédie enseigne. [...] La reconnaissance de soi est au prix d'un dur apprentissage acquis au cours d'un long voyage à travers ces conflits persistants, dont l'universalité est inséparable de leur localisation chaque fois indépassable.» (1990, p. 283)

La rencontre de situations tragiques nous oblige à un difficile travail de déchiffrage, précisait Ricoeur lors d'une entrevue (1995, p. 142): «la rencontre de situations tragiques; c'est là que j'affronte toutes les perplexités: le soi sans le soutien d'une constitution de mêmeté, avec la question: Comment déchiffrer sa propre vie dans les situations d'incertitude, de conflit ou de risque? » Nous apprenons à dire «me voici!» en nous confrontant à une énigme, en tentant de déchiffrer notre propre vie.

Comment déchiffrer ce qui s'est passé lors de l'irruption du tragique, lors d'un suicide, par exemple? On l'oublie souvent, mais il nous est possible d'avoir accès à des tentatives de déchiffrage de ce vécu tragique grâce à l'œuvre littéraire et à l'analyse littéraire. En relisant quelques-uns des précédents numéros de Frontières, on trouve des articles qui s'attachent à le faire, comme celui de Catherine Mavrikakis (2002, p. 24-26) qui commente deux textes d'Hubert Aquin dans lesquels le narrateur se prépare à son suicide, et celui de Lorca Hassan Almohammed (2007, p. 41-46) qui nous invite à accompagner avec lui la «marche à la mort» du poète français Olivier Larronde.

Frontières a souvent publié les témoignages d'écrivaines et d'écrivains qui, comme Ricoeur, ont perdu de façon tragique quelqu'un qui leur était cher. Il faut relire ces textes d'une lucidité poignante, par exemple le texte intitulé «Claude est mort sans m'en parler», écrit par Denise Blais (2003, p. 76-77). Sous la plume de Michaël La Chance (2001), la tragédie est déchiffrée sous la forme d'une corrida, « une corrida pour soi seul», écrit-il dans un texte dédié à son ami Jacques Sanchiz:

Jouer une corrida pour soi seul : c'est la seule façon de ramener sa vie dans un éclair de dignité. C'est perdre la vie mais la rendre à elle-même, à la dignité à laquelle elle a droit. [...] La plupart étirent leur vie, le plus longtemps possible, comme s'ils en savaient quelque chose. Combien de temps peut-on opposer la franchise à l'hébétude? Tous ceux qui croient d'emblée posséder ce qu'ils sont, pour ensuite être ce qu'ils ont, se méfient de ceux qui ne possèdent pas, qui ne peuvent ajuster le pas et titubent. (2001, p. 60)

Ceux qui avancent sans tituber sont parfois dans l'hébétude plutôt que dans la franchise. Ce sont aussi ceux-là qui «s'en vont forts de leur vie », comme le décrivait Saint-Denys Garneau dans l'extrait cité en exergue. Ceux-là ont le cœur «dur» ajoutait le poète. Nous qui observons ceux qui titubent, nous qui étudions le phénomène du suicide dans l'espoir de trouver des modes d'intervention pour le prévenir, accepterons-nous de nous

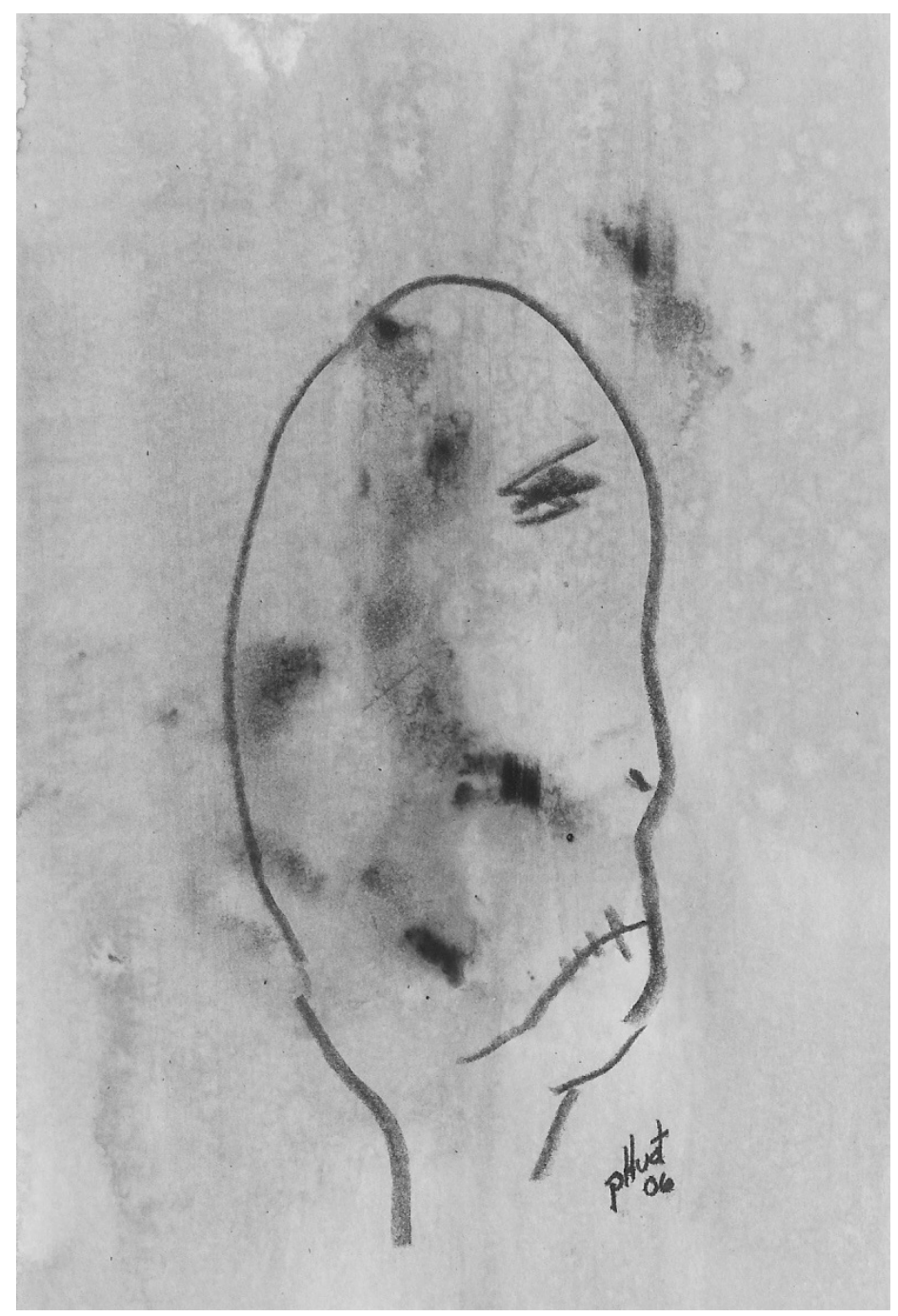

observer nous-mêmes, au risque de nous reconnaître en danger d'hébétude, au risque de constater que nous faisons partie de ceux qui ont le cœur "dur» face à ceux dont le cœur est «déjà tout en sang »? Un poète peut nous poser la question; qui d'autre oserait?

La littérature balise ici des chemins dont l'accès est difficile. Mais quand viendra le temps de créer des programmes pour prévenir le suicide, découvrirons-nous que les enseignements tirés de la philosophie et de la littérature sont incompatibles avec l'approche que privilégient les approches quantitatives? Devrons-nous conclure que notre réflexion sur «le tragique de l'action » est un luxe inutile, qui risque de ralentir indûment le travail à faire à partir de résultats mesurables? Nous savons qu'il faut agir efficacement; nous avons souvent peur de ne pas agir assez vite.

\section{PARTIR EN PEUR, CE SERAIT OUBLIER LA COMPLÉMENTARITÉ DES DISCIPLINES}

Il faut prévenir le suicide et il y a urgence de le faire. Mais on ne saurait y arriver en lançant des programmes à la hâte ou en prenant des décisions prématurées ${ }^{10}$. Toutes les ressources de l'interdisciplinarité devront plutôt être mises à contribution pour que nous en arrivions à intervenir avec le doigté nécessaire.

Le défi à relever consiste entre autres à bâtir des ponts entre les disciplines dites «scientifiques» et les sciences dites « humaines». Ce travail est déjà amorcé, mais il rencontre encore des obstacles que plusieurs déplorent. À cet égard, il est utile de relire un article bien documenté, publié dans Frontières en 2003 et intitulé: «Ces 
pédopsychiatres que T. et Y. n'ont pas consultés». Dans ce texte, trois chercheurs décrivent ce qu'ils ont observé dans le cadre d'une recherche menée auprès des intervenants en prévention du suicide dans les centres jeunesse. Ils ont jugé nécessaire d'exposer comment "l'intervention des éducateurs en matière de risques suicidaires a été influencée, voire même transformée par deux suicides d'adolescents survenus en 1994 et 1997, suivis d'une série de rapports d'enquêtes, d'autopsies psychologiques et de protocoles d'intervention » (Lafortune, Deslauriers-Varin et Bouchard, 2003, p. 78).

\section{C'EST L'IMMENSE PHÉNOMÈNE QUE CONSTITUE}

LE FAIT D'ÊTRE VIVANT QUI DEMEURE

\section{ULTIMEMENT L'OBJET DE TOUTE RECHERCHE}

\section{ET DE TOUTE PRATIQUE; IL NOUS REVIENT}

\section{DE CHOISIR ALORS D'INSCRIRE NOTRE DÉMARCHE}

\section{DANS UNE VISÉE DE LA VIE BONNE.}

Le programme de recherche de ces auteurs portait sur le recours à la psychopharmacothérapie dans la prise en charge des jeunes placés dans des centres jeunesse. Après avoir mené des entretiens semi-directifs avec des éducateurs en centres jeunesse en 2001 et 2002, ils constatent que malgré les exhortations à l'interdisciplinarité formulées lors de l'introduction d'un nouveau protocole d'intervention liant les centres jeunesse aux milieux médicaux, "aucun modèle intégratif » n'a vraiment été proposé (p. 83) et les tensions sont visibles:

Des tensions conceptuelles risquent de s'inscrire autour des clivages corps/esprit, démarche scientifique/démarche humaniste, traitement relationnel dit «naturel»/ traitement pharmacologique dit «chimique». La formation médicale peut s'appuyer parfois sur un organicisme strict, selon lequel «les maladies mentales sont des maladies du cerveau ». De la même façon, les formations humaniste, cognitivo-behaviorale ou psychanalytique, peuvent avoir du mal à prendre en compte réellement la dimension organique, les neurotransmetteurs, les synapses et les structures anatomiques. (p. 84)

Ces observations datent de 2001-2002 et les auteurs se réfèrent à un rapport publié en 1999... mais le message garde une actualité déroutante pour le lecteur d'aujourd'hui. Force est de constater que les chasses gardées et les cloisonnements disciplinaires ne sont pas disparus et que le dialogue entre les spécialistes du modèle médical et ceux des sciences humaines donne rarement lieu à des éclairages réciproques :

Les auteurs du rapport sur le suicide déplorent les chasses gardées et les cloisonnements disciplinaires mais ils n'offrent aucune issue au plan conceptuel, si ce n'est un scénario où tous se rallieraient au modèle bio-psycho-social. Or il est difficile d'oublier que ce modèle provient de la psychiatrie, au même titre que le DSM-IV. Faut-il ici comprendre qu'aux yeux du Comité, le modèle intégrateur et rassembleur des diverses professions devrait être le modèle médical? (p. 84)

Les idées maîtresses du rapport dont parlent ici Lafortune, Deslauriers-Varin et Bouchard (p. 79) font l'éloge de la complémentarité des disciplines. Mais, constatent les chercheurs, le rapport passe sous silence le caractère indispensable d'une «interrogation réciproque sur le niveau de connaissance des problématiques » atteint, avec des outils différents et selon des voies plurielles, par chacune des disciplines.

Lafortune, Deslauriers-Varin et Bouchard commentent, entre autres, le recours à l'autopsie psychologique (p. 81, 83-84). Or l'article de Vrakas, dans le présent numéro, décrit une recherche récente réalisée en utilisant cette méthodologie ${ }^{11}$. Après avoir lu en parallèle ces deux textes, on peut mesurer l'ampleur du défi qui consisterait, par exemple, à exploiter en complémentarité les deux notions d'autopsie psychologique et d'attestation de soi. La notion d'autopsie psychologique renvoie à des expertises médicales; la notion d'attestation de soi que nous venons d'explorer avec Ricoeur s'inscrit dans des réflexions philosophiques. Les tenants de la première ne connaissent peut-être même pas l'existence de la seconde, et vice versa. Les nombreuses disciplines qui peuvent être mises à contribution dans les recherches visant à prévenir le suicide ont encore beaucoup à découvrir l'une au sujet de l'autre dans le cadre d'échanges réciproques. La complémentarité des disciplines ne viendra qu'après cet apprivoisement mutuel; elle exigera des chercheurs et des praticiens un «dégrisement du Même enivré de soi ${ }^{12}$ », pour reprendre une expression qu'utilise Lévinas dans La mort et le temps (1991, p. 25).

Dans son «Interlude»sur «le tragique de l'action», Ricoeur rappelle à la philosophie, à l'éthique et à la morale - et du même coup à toutes les autres disciplines qui veulent traiter du tragique d'une existence humaine - qu'elles n'y trouveront pas, comme le tailleur de pierres qui se rend dans une carrière, des matériaux qui attendent passivement qu'on les exploite avec compétence. Même avec une expertise pointue, on ne refaçonne pas à sa guise le tragique de l'action humaine:

Que le tragique résiste à une «répétition» intégrale dans le discours de l'éthique et de la morale, ce trait doit être rappelé avec brièveté, mais avec fermeté, de peur que la philosophie ne soit tentée de traiter la tragédie à la façon d'une carrière à exploiter, d'où elle tirerait les plus beaux blocs qu'elle retaillerait ensuite à sa guise souveraine. (1990, p. 281)

Domaines d'expertise et champs de recherches subventionnées sont des lieux qui nous sont plus familiers que la carrière de pierres dont parle Ricoeur, mais la mise en garde vaut quand même pour le chercheur ou l'intervenant quelle que soit sa discipline: le tragique de l'attestation soi n'est pas un matériau que l'on peut retailler à sa guise. Déchiffrer sa propre vie pour en arriver, malgré le tragique de la situation, à une attestation de soi plus fiable, c'est - Ricoeur nous a déjà prévenus - un «dur» apprentissage. La souffrance fait partie de l'équation à résoudre. Pour Ricoeur, l'attestation se définit, répétons-le, «comme l'assurance d'être soi-même agissant et souffrant» (1990, p. 35).

Or, peut-on vraiment ramener la souffrance à un simple objet d'étude? Dans les milieux d'intervention, le praticien est constamment confronté à la souffrance. «Quelle place faisonsnous à la souffrance de l'être? ", c'est une question qui se pose avec une acuité tragique dans le cas du suicide, comme le soutient éloquemment Natacha Joubert, une intervenante qui, en 2002, donne un titre percutant à l'article qu'elle publie dans Frontières : «La prévention du suicide au Québec. À tant vouloir éviter la mort, on finit par éviter la vie». Ce texte fait état d'un constat troublant:

Nulle part ailleurs la souffrance de l'être effraie autant qu'au sein de disciplines scientifiques comme la médecine, la psychiatrie ou la suicidologie qui la côtoient pourtant tous les jours, mais qui ont le plus souvent contribué, à travers leur propre fuite, à nous en éloigner. Ainsi la souffrance de l'être est rarement discutée en dehors des nomenclatures médicales ou psychiatriques, c'est-à-dire qu'elle est le plus souvent 
liée ou enfermée dans un diagnostic. Ici le souci d'être rigoureux et scientifique sert le déni pas le progrès. (p. 56) Ce qui fait peur, dit Natacha Joubert, c'est «la souffrance de l'être ${ }^{13} »$. Partir en peur, en oubliant la complémentarité des disciplines, ce serait passer à côté de cette souffrance. La posture du chercheur et aussi celle du praticien les oblige à maints égards à se donner ce cœur «dur» dont parlait Saint-Denys-Garneau. Mais le poète ajoute cette remarque paradoxale sur le cœur des vivants : il est «dur comme un bon arbre». Les vivants sont «forts de leur vie». C'est l'immense phénomène que constitue le fait d'être vivant qui demeure ultimement l'objet de toute recherche et de toute pratique; il nous revient de choisir alors d'inscrire notre démarche dans une visée de la vie bonne. Ricoeur fait d'ailleurs de cette visée le premier moment de l'avancée en trois temps qui lui permet de proposer une définition de l'éthique ${ }^{14}$.

Pour «attester» de sa compétence en prévention du suicide, le chercheur ou le praticien, quel que soit son champ disciplinaire, voudra se retenir de partir en peur car ce serait nier le tragique de l'attestation de soi et oublier la complémentarité des disciplines. Chercheurs ou praticiens, nous ne sommes pas à l'abri de tout soupçon. Mais, nous l'avons vu avec Ricoeur, «le soupçon est aussi le chemin vers et la traversée dans l'attestation» (1990, p. 350). Attester de soi, c'est, dit-il, relever l'épreuve du soupçon pour en arriver à une attestation de plus en plus fiable. Avec ses trois numéros sur le thème du suicide, Frontières apporte sa contribution à l'émergence d'une attestation de plus en plus fiable des compétences interdisciplinaires sur le phénomène du suicide.

\section{Bibliographie}

ALMOHAMMED, L.H. (2007). «Olivier Larronde ou la mache à la mort», Frontières, vol. 19, nº 2, p. 41-46.

BLAIS, D. (2003). «Claude est mort sans m'en parler», Frontières, vol. 15, $\mathrm{n}^{\circ}$ 2, printemps, p. 76-77.

DUHAMEL, A. (2003). "Le tragique comme catégorie d'analyse éthique », dans Lacroix, A. et Malherbe, J.F. (dir.), L'éthique à l'ère du soupçon, Montréal, Liber, p. 115-130.

GAGNÉ, M. et D. ST-LAURENT (2009). La mortalité par suicide au Québec: tendances et données récentes - 1981 à 2007, l'Institut national de santé publique du Québec, Direction recherche, formation et développement, $\mathrm{n}^{\circ}$ de publication 890. Ce document est disponible intégralement en format électronique (PDF) sur le site Web de l'Institut national de santé publique du Québec au: http://www.inspq.qc.ca.

JOUBERT, N. (2002). «La prévention du suicide au Québec. À tant vouloir éviter la mort on finit par éviter la vie», Frontières, vol. 15, $\mathrm{n}^{\circ} 1$, automne, p. 52-59.

LA CHANCE, M. (2001). «Corrida pour soi seul », Frontières, vol. 14, $\mathrm{n}^{\circ} 1$, automne, p. 59-62.

LAFLAMME, D. (2008). «Ethics and the interplay between the logic of the Excluded middle and the logic of the Included middle», dans Nicolescu, B. (dir.), Transdisciplinarity: Theory and Practice, New Jersey, Hampton Press, p. 147-154.

LAFORTUNE, D., N. DESLAURIERS-VARIN et R. BOUCHARD (2003). "Ces pédopsychiatres que T. et Y. n'ont pas consultés», Frontières, vol. 16, $\mathrm{n}^{\circ} 1$, automne, p. 78-84.

LÉVINAS, E. (1991). La mort et le temps, Paris, L'Herne.

MAVRIKAKIS, C. (2002). "La mort en quatrième vitesse. Ou comment annoncer sa fin pour tenter d'en finir », Frontières, vol. 14, n 2 , automne, p. 24-28.

RICOEUR, P. (1990). Soi-même comme un autre, Paris, Seuil.

RICOEUR, P. (1991). «L'attestation : entre phénoménologie et ontologie », dans Greich, S. et R. Kearny (dir.), Paul Ricoeur. Les métamorphoses de la raison herméneutique, Paris, Cerf, p. 381-403.

RICOEUR, P. (1995). La critique et la conviction. Entretien avec François Azouvi et Marc de Launay, Paris, Calmann-Lévy.
SAINT-DENYS-GARNEAU, H. DE (1949). Poésies complètes, Ottawa, Fides.

SOCIÉTÉ DU PARLER FRANÇAIS AU CANADA (1930). Glossaire du parler français au Canada, Québec, L’Action sociale.

\section{Notes}

1. «Prendre peur, prendre l'épouvante», selon le Glossaire du parler français au Canada publié en 1930 par la Société du parler français au Canada, p. 511.

2. Au sujet de l'ancrage de cette symbolique, on peut lire dans ce numéro le texte de Louis-Vincent Thomas; à la page 142, au début de la section intitulée "Le suicide et l'Occident aujourd'hui », il fait référence à des enquêtes qu'il a menées sur ce qu'il appelle «la mort atomique».

3. Nous reproduisons dans la section Archives du présent numéro un extrait d'un texte portant sur les suicides que le regretté Louis-Vincent Thomas a publié en 1988 dans le volume 1, numéro 2 de Frontières. Ce numéro est maintenant épuisé.

4. Nous reproduisons à la page 140 la Table des matières de ce numéro de Frontières, qui a fait l'objet d'une réimpression pour répondre à la demande.

5. «Depuis le sommet de 22 décès par 100000 personnes atteint en 1999, le taux de suicide a diminué en moyenne de 4,1\% par année» entre 1999 et 2007 (Gagné et St-Laurent, 2009, p. 1). Pour une analyse de la période 1981 à 2005, voir l'article de ces auteurs dans le présent numéro, à la page 44.

6. La logique du tiers inclus de Séphane Lupasco est un exemple de logique non binaire. Voir à ce sujet, Laflamme, 2008, «Ethics and the Interplay between the Logic of the Excluded Middle and the Logic of the Included Middle», p. 147 à 154.

7. Paul Ricoeur et les soldats de l'unité qu'il commandait ont été faits prisonniers en mai 1940 ; il a passé cinq ans dans un camp de prisonniers en Allemagne.

8. Sur l'attention au tragique chez Paul Ricoeur, voir Duhamel, A. (2003).

9. Ricoeur précise qu'il se réfère plus particulièrement à la tragédie grecque et il choisit comme exemple la tragédie d'Antigone qu'il commente longuement. Notons que le présent numéro contient également, dans la section Hors thème, un texte portant sur les tragiques grecs. Il s'agit d'un article de Essam Safty, intitulé: «La polyvalence du moi tragique. La portée idéologique des dernières volontés chez les tragiques grecs ». Safty n'aborde pas directement la thématique de la prévention du suicide mais s'intéresse plutôt aux dernières volontés, vœux, invocations et imprécations des mourants; le texte contient aussi de brèves allusions aux «suicides d'amour».

10. L'article de Julien et Breton, dans ce numéro signale clairement ce danger en ce qui concerne la prévention du suicide chez les adolescents. Voir p. 23.

11. Notons que dans le présent numéro, l'article de Tousignant et al. fait aussi référence à une «étude par autopsie psychologique » réalisée en milieu autochtone. Voir p. 113.

12. Cet énoncé de Lévinas est immédiatement suivi des phrases suivantes: "Ce éveil doit être pensé comme éveil dans l'éveil : l'éveil comme tel devient un état - il faut donc un éveil de cet éveil. Il y a une itération de l'éveil» (p. 25). Ce que le philosophe propose ici correspond à ce que la théorie des systèmes autoréférentiels appelle une observation de second degré, c'est-à-dire une observation de sa propre observation, faite par le système-observateur lui même.

13. Le vocabulaire de l'intervention rejoint ici celui de la philosophie, cette dernière n'ayant pas l'exclusivité lorsqu'il s'agit de donner sens au mot être, comme l'explique Lévinas (1991, p. 26-27): «Le verbe être est d'ores et déjà, avant toute ontologie explicite, compris par les hommes. Il est compris préontologiquement et donc sans entente pleine, mais au contraire avec subsistance de questions. [...] Il faut donc s'approcher du sens de l'être à partir de l'homme qui est un étant comprenant l'être et questionnant, s'interrogeant sur l'être. »

14. Ricoeur définit l'éthique comme «la visée de la vie bonne avec et pour autrui dans des institutions justes» (1990, p. 202). 